\title{
Tecnura
}

\section{Revisión literaria y modelación de la desaturación de un suelo arenoso por medio del método de inyección de aire}

\section{Literature Review and Modelling of the Desaturation of a Sandy Soil by Means of the Air Injection Method}

\author{
Sebastián Sepúlveda-Cano (iD) ${ }^{1}$, Carlos Alberto Vega-Posada (iD)2, Edwin Fabián \\ García-Aristizábal iD3
}

Fecha de Recepción: 06 de Agosto de 2020

Fecha de Aceptación: 24 de Febrero de 2021

Cómo citar: Sepúlveda-Cano., S. Vega-Posada., C.A. y García-Aristizábal., E.F. (2021). Revisión literaria y modelación de la desaturación de un suelo arenoso por medio del método de inyección de aire. Tecnura, 25(68), 140-164. https:/ / doi.org/10.14483/22487638.16282

\section{Resumen}

Contexto: En los últimos años, la comunidad científica ha discutido la posibilidad y la efectividad de disminuir el grado de saturación del suelo como una medida para mejorar la resistencia mecánica de las arenas saturadas licuables. Por esta razón, se han propuesto y probado varias metodologías para introducir burbujas de gas en el suelo. Además, es bien sabido que la disminución del grado de saturación del suelo, al inducir una pequeña cantidad de aire, restringe el desarrollo de exceso de presión de poros y aumenta significativamente su resistencia cíclica.

Método: Se proporciona una visión general de la tecnología de inyección de aire para inducir un grado de saturación parcial, mediante la selección de algunos artículos de alta calidad y la revisión literaria sistemática. Asimismo, se desarrolla un modelo de flujo multifásico y se lleva a cabo un análisis numérico para describir la evolución, en el tiempo y en el espacio, de la desaturación del suelo, para lo cual se investiga numéricamente la longitud del avance del frente de aire durante la inyección.

Resultados: Se reconoce hacia dónde se dirige la investigación futura relacionada con la inyección de aire, como medida de mejoramiento de suelos arenosos licuables. Además, se entrega un estudio paramétrico transitorio evaluando la permeabilidad del suelo, inyectabilidad de aire, presiones de inyección, y radio de desaturación de la región de interés.

Conclusiones: El trabajo destaca hallazgos e identifica enfoques de investigadores, mediante observaciones, para lo cual se reconocen limitaciones y lagunas de conocimiento. Finalmente, los resultados numéricos indican que la evolución de la desaturación está controlada activamente por la presión de inyección de aire y la permeabilidad del suelo.

Palabras clave: desaturación de suelo, licuefacción, mejoramiento de suelos.

\footnotetext{
${ }^{1}$ Ingeniero Civil, magíster en Ingeniería (c), Escuela Ambiental, Facultad de Ingeniería, Universidad de Antioquia, Medellín, Colombia. Contacto: sebastian.sepulveda@udea.edu.co

${ }^{2}$ Ingeniero civil, magíster en Ingeniería, PhD. Profesor asociado, Escuela Ambiental, Facultad de Ingeniería, Universidad de Antioquia, Medellín, Colombia. Contacto: carlosa.vega@udea.edu.co

${ }^{3}$ Ingeniero civil, magíster en Ingeniería, PhD. Profesor titular, Escuela Ambiental, Facultad de Ingeniería, Universidad de Antioquia, Medellín, Colombia. Contacto: edwin.garcia@udea.edu.co
} 


\begin{abstract}
Context: In recent years, the scientific community has discussed the possibility and effectiveness of decreasing the degree of soil saturation as a measure to improve the mechanical resistance of liquefiable saturated sands. For this reason, several methodologies have been proposed and tested to introduce gas bubbles into the soil. Furthermore, it is well known that decreasing the degree of soil saturation by inducing a small amount of air restricts the development of excess pore pressure and significantly increases the cyclic resistance of the soil.

Methodology: An overview of air injection technology to induce a degree of partial saturation is provided by selecting high quality articles through a systematic literature review. In addition, a multiphase flow model is developed and a numerical analysis is carried out to describe the evolution, in time and space, of the desaturation of the soil, investigating numerically the length of the air front advance during injection.

Results: It is recognized where future research related to air injection as a measure of liquefiable sandy soil improvement is moving. In addition, a parametric transient study is provided evaluating soil permeability, air injectivity, injection pressures, and desaturation rate of the region of interest.

Conclusions: The study highlights findings and identifies researcher approaches, making observations, recognizing limitations and knowledge gaps. Finally, the numerical results obtained indicate that the evolution of desaturation is actively controlled by air injection pressure and soil permeability.
\end{abstract}

Keywords: liquefaction, soil improvement, soil desaturation.

\title{
Tabla de Contenidos
}

Página

INTRODUCCIÓN

EFECTO DEL GAS EN LA RESISTENCIA A LA LICUEFACCIÓN

INYECCIÓN DE AIRE PARA INDUCIR GRADO DE SATURACIÓN

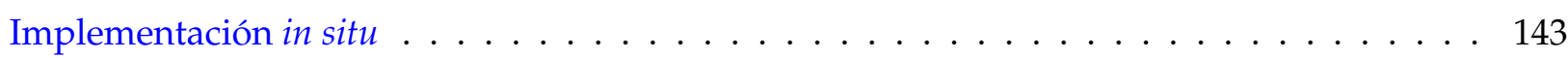

Longevidad del efecto de inyección de aire . . . . . . . . . . . . . . . . 145

Evalución de área desaturada y radio de influencia . . . . . . . . . . . . . . . . . . . . . . . . . . . . . . . . . . . . . . . . . . . . . . . .

Presión de inyección . . . . . . . . . . . . . . . . . . . . 146

$\begin{array}{ll}\text { Experimentación } & 147\end{array}$

$\begin{array}{lr}\text { Modelación numérica } & 148\end{array}$

$\begin{array}{ll}\text { MODELO MATEMÁTICO } & 149\end{array}$

ANÁLISIS Y MODELACIÓN DE INYECCIÓN DE GAS EN MATERIAL SATURADO 151

Validación del modelo propuesto . . . . . . . . . . . . . . . . . . . . . 151 
Análisis paramétrico de inyección de aire . . . . . . . . . . . . . . . . . . 153

$\begin{array}{ll}\text { CONCLUSIONES } & 156\end{array}$

$\begin{array}{lc}\text { REFERENCIAS } & 157\end{array}$

\section{INTRODUCCIÓN}

La mayoría de las técnicas de mejoramiento de suelos susceptibles a licuefacción requieren un gran gasto energético debido a los procesos de producción y de construcción in situ. Existe una clara necesidad de desarrollar técnicas de mitigación de licuefacción rentables y aplicables sobre estructuras nuevas y existentes (Gallagher, Pamuk y Abdoun, 2007), por lo que científicos plantean la inducción parcial del grado de saturación como una de ellas (Okamura, Ishihara y Tamura, 2003). Este proceso radica en la introducción de burbujas de gas en el suelo, que provocan una disminución del módulo volumétrico y un aumento de la compresibilidad de la mezcla aire y agua dentro de los poros, de modo que cuando el suelo está sujeto a cargas cíclicas, el gas absorbe el exceso de presión de poros reduciendo su volumen (Okamura y Soga, 2006), lo que resulta en un aumento de la resistencia a la licuefacción. Algunos métodos propuestos para generar burbujas son la inyección de aire (Ishihara, Okamura y Oshita, 2003), la electrólisis del agua (Yegian, Eseller-Bayat, Alshawabkeh y Ali, 2007), las reacciones químicas (Eseller-bayat, Yegian y Alshawabkeh, 2013) y los procesos microbiológicos (He, Chu e Ivanov, 2013), entre otros. Estos métodos son menos invasivos, rentables para áreas extensas y prácticos cuando el tratamiento puede afectar la capacidad admisible de los cimientos durante el procedimiento (Marasini y Okamura, 2015a).

En ese sentido, este documento tiene como propósito presentar una revisión bibliográfica sobre la desaturación de suelos licuables mediante el proceso de inyección de aire, enfatizándo en los aspectos prácticos y consideraciones sobre la geotecnia de los suelos tratados. Además, se plantea una modelación numérica, considerando un flujo bifásico incompresible e isotérmico en medios porosos isotrópicos homogéneos con efectos capilares, para evaluar el efecto de la presión de inyección de aire y la permeabilidad del suelo sobre el radio de desaturación, mediante un análisis paramétrico a través del software FlexPDE, con su conFiguración gratuita (PDE Solutions Inc., 2005), que proporciona herramientas para la solución de ecuaciones diferenciales parciales.

\section{EFECTO DEL GAS EN LA RESISTENCIA A LA LICUEFACCIÓN}

En los últimos años se ha estudiado la desaturación del suelo y su influencia en la susceptibilidad a la licuefacción (Tsukamoto, Kawabe, Matsumoto y Hagiwara, 2014). Algunos de los estudios más significativos que muestran el efecto de la desaturación del suelo en el incremento de su resistencia se describen a continuación: según pruebas cíclicas informadas por (Yang, Savidis y Roemer, 2004, 
Tsukamoto et al., 2002), y (Bouferra, Benseddiq y Shahrour, 2007), se define que la reducción en el grado de saturación puede resultar en un aumento significativo en la resistencia a la licuefacción. Por otro lado, (Ishihara, Huang y Tsuchiya, 1998) demuestran que la rigidez volumétrica de los fluidos varía con la inclusión de un pequeño volumen de gas, afectando la respuesta de la presión de poros, el valor del parámetro B de Skempton, la onda P y la resistencia cíclica. Sobre la respuesta en presión de poros, (Yang, Savidis, Sato y Li, 2003) muestran que una reducción de la saturación en un 1\%, implica una reducción en la proporción de exceso de presión de poros de 0,6 a 0,15 bajo excitación horizontal pura. Además, (Rad, Vianna y Berre, 1994) encontraron que la arena con gas no soluble es más resistente que la que contiene gas soluble; igualmente, el modelo definido por (Grozic, Nadim y Kvalstad, 2005) permite concluir que la resistencia al corte no drenado del suelo gaseoso aumenta con una reducción en el grado de saturación.

Asimismo, (Grozic, Robertson y Morgenstern, 1999) indican que, bajo compresión no drenada, la arena suelta muestra una transición del ablandamiento al endurecimiento por deformación para grados de saturación menores a $90 \%$. Por otra parte, sobre pruebas cíclicas con un grado de saturación del $75 \%$ al 99\%, el gas aumenta la resistencia cíclica del $200 \%$ al $300 \%$. La Figura 1 muestra una recopilación de datos experimentales tomados de varias fuentes literarias donde se muestra el aumento de la relación de resistencia a la licuefacción a medida que disminuye el grado de saturación $\left(S_{w}\right)$, presentando diferentes variaciones de acuerdo con el tipo de suelo ensayado, lo que indica la incidencia de otros factores sobre la respuesta de las arenas ante cargas dinámicas.

En la Figura 1 se unificaron algunos tipos de suelo y se definieron las líneas de regresión para arena de Urayasu (línea verde / $R^{2}=0,87$ ), arena de Toyoura (líneas negras / $R^{2}=0,95-0,99$ ), arena de Niigata (línea roja / $R^{2}=1,00$ ) y arena Inagi (líneas azules / $R^{2}=0,55$ y 0,92). Los resultados obtenidos indican que se explica alguna porción de la variabilidad de los datos en respuesta a sus valores medios. Se observa que los datos con mayor dispersión respecto de su media, ordenados de mayor a menor, son los asociados a la Arenas Inagi, Urayasu, Toyoura y Niigata, respectivamente. Además, se logra percibir que la saturación de la fase líquida se asocia fuertemente en un sentido inverso con la relación de resistencia a la licuefacción.

\section{INYECCIÓN DE AIRE PARA INDUCIR GRADO DE SATURACIÓN}

\section{Implementación in situ}

Algunos autores como (Okamura et al., 2011, Okamura y Tomida, 2015), y (Tomida, 2014) han implementado esta técnica para mejorar la resistencia a la licuefacción de arenas. En las pruebas in situ presentadas por (Okamura et al., 2011), el flujo de aire se aplica desde el inyector a un estrato licuable hasta alcanzar una extensión de desaturación parcial de $4 \mathrm{~m}$ medidos horizontalmente con respecto al inyector, de lo que se obtiene una variación del grado de saturación $\left(S_{w}\right)$ entre 68 \% y 98 \%. Poste- 


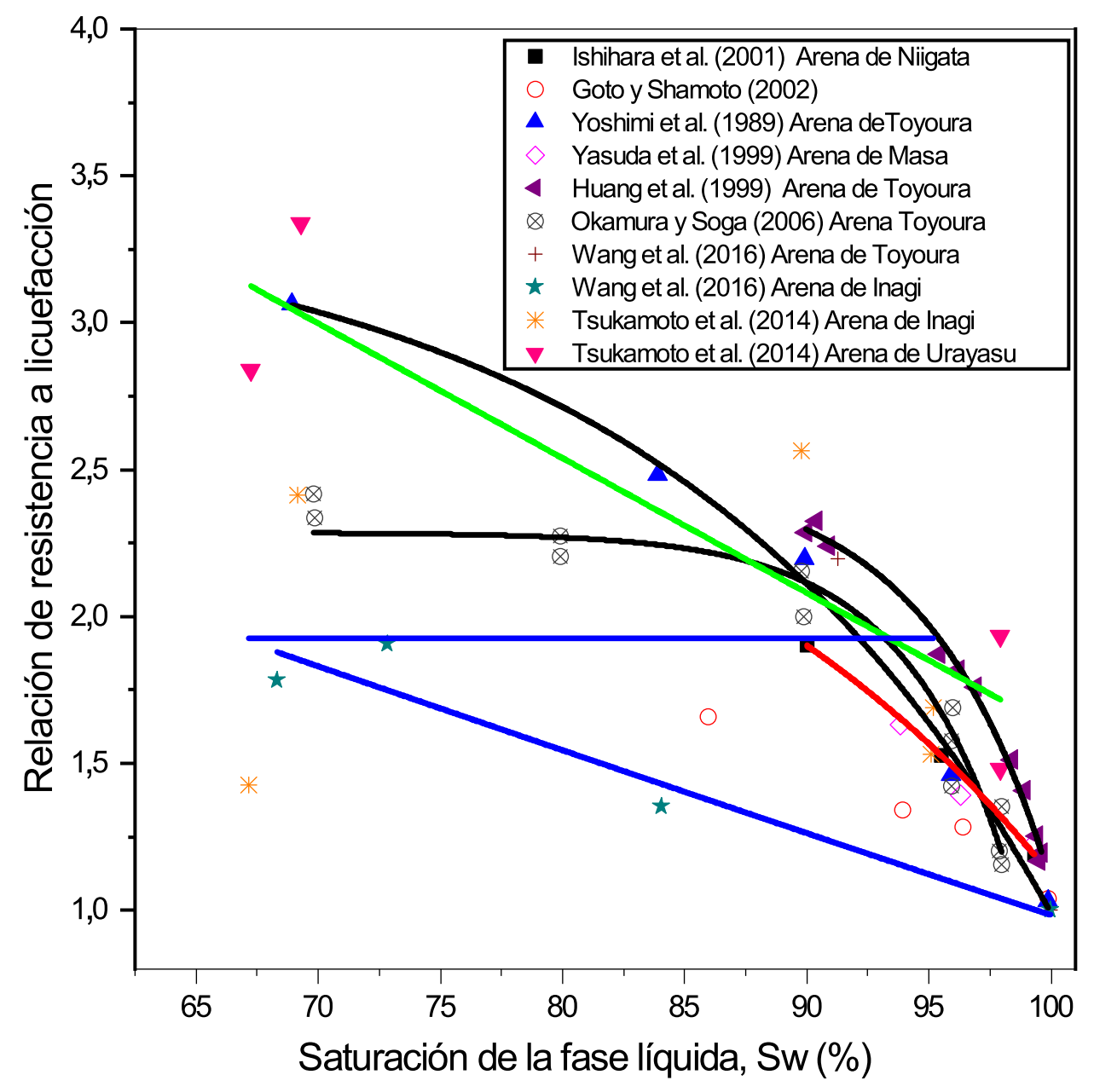

Figura 1. Relación entre $S_{w}$ y la relación de resistencia a la licuefacción definida por el criterio de deformación axial de doble amplitud del $5 \%$ después de 20 ciclos de carga (DA = 5\%)

Fuente: elaboración propia.

riormente, los autores realizaron las pruebas cíclicas cuyos resultados indicaron que la resistencia a la licuefacción se duplica. Los mismos autores mencionan que los materiales utilizados son de fácil adquisición, sin necesidad de ningún equipo especializado para su diseño e implementación.

La técnica de desaturación in situ consiste en la inserción de una tubería en los estratos de suelos licuables por donde es inyectado el aire a presión, como se muestra en la Figura 2. Entre las ventajas de este método se destaca la poca perturbación para las operaciones de superficie, la eficiencia, el corto tiempo de ejecución, el bajo costo, la aplicabilidad en zonas de difícil acceso y la posibilidad de integración con otras tecnologías para aumentar la eficiencia; igualmente, no implica la extracción, el tratamiento, el almacenamiento o la liberación de agua subterránea. Información extrapolable de 


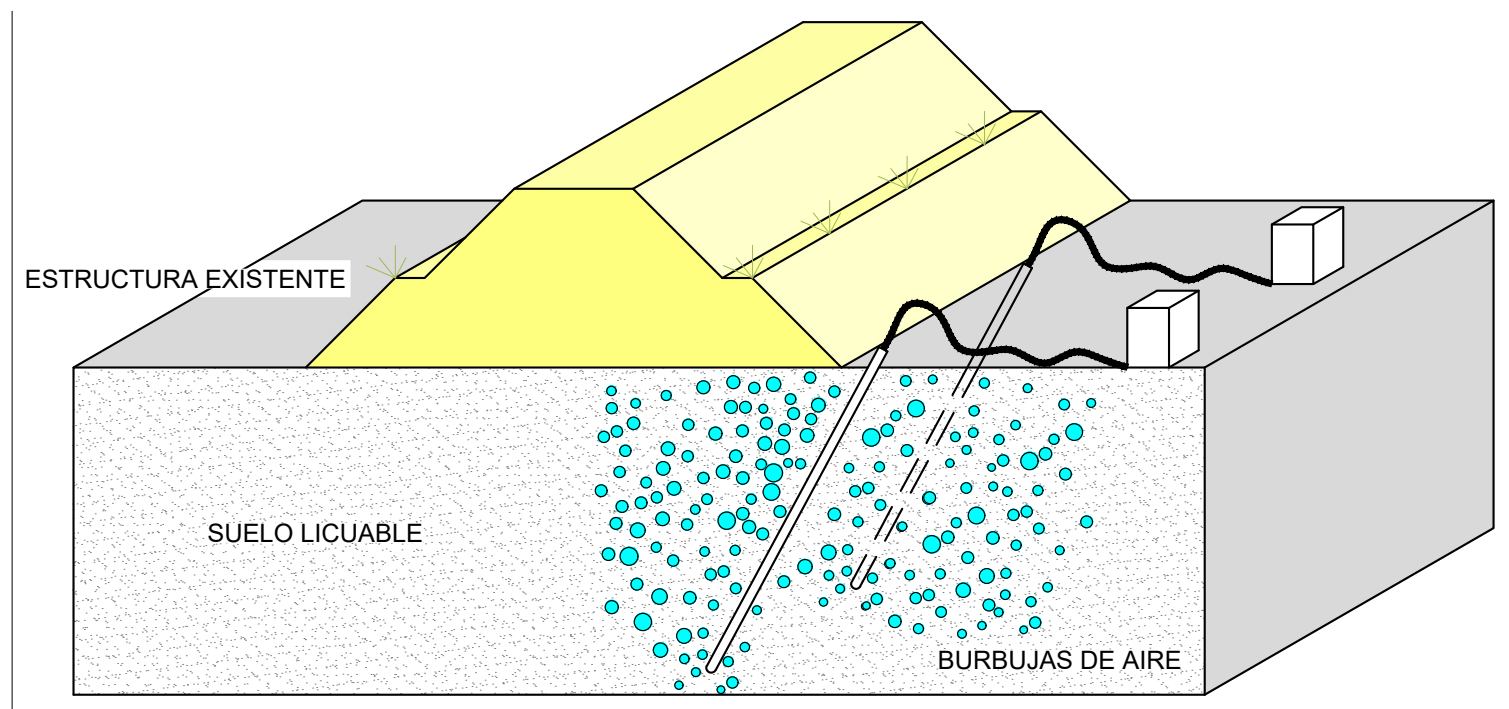

Figura 2. Desaturación por inyección de aire

Fuente: modificada de (Okamura y Teraoka, 2005b).

la técnica de volatilización in situ, sobre las especificaciones y operación que puede llegar a fortalecer los desarrollos de la inducción parcial del grado de saturación mediante inyección de aire, se encuentra en (US EPA, 1992, Wisconsin DNR, 1995, Holbrook et al., 1998).

\section{Longevidad del efecto de inyección de aire}

El área desaturada y el radio de influencia se han estudiado mediante pruebas centrífugas: un ejemplo de esto es el trabajo de (Okamura y Tamura, 2004) donde investigan zonas que fueron desaturadas anteriormente (4, 8 y 26 años atrás). Las pruebas de laboratorio demostraron que para la zona mejorada hace 26 años todavía existían burbujas de aire; además, ninguna de las muestras de las zonas tenía más del $90 \%$ de $S_{w}$. En un estudio similar, (Okamura y Teraoka, 2005a) observaron aumentos del $10 \%$ para $S_{w}$, lo que indica longevidad de las burbujas inyectadas, por lo que generar grados de saturación por debajo del $90 \%$ produce condiciones de desaturación duraderas. Estudios reconocen que la longevidad del aire dentro del suelo típicamente es mayor a los diez años (Okamura, Ishihara y Tamura, 2006,Okamura et al., 2009,Vega, Ramos y García, 2017). (Zeybek y Madabhushi, 2017c) estudiaron la durabilidad de las burbujas en condiciones hidrostáticas, a baja y alta presión de poros, flujo vertical ascendente y descendente, presión de poros variable y agitación lateral. Su estudio revela que el volumen de burbujas ocluidas arrastradas por el flujo de agua a través de la matriz del suelo es mínimo y que la mayoría de las burbujas permanecen atrapadas en los poros del suelo. Los resultados incluso respaldan los mostrados por (Eseller-bayat, Yegian y Alshawabkeh, 2013). 


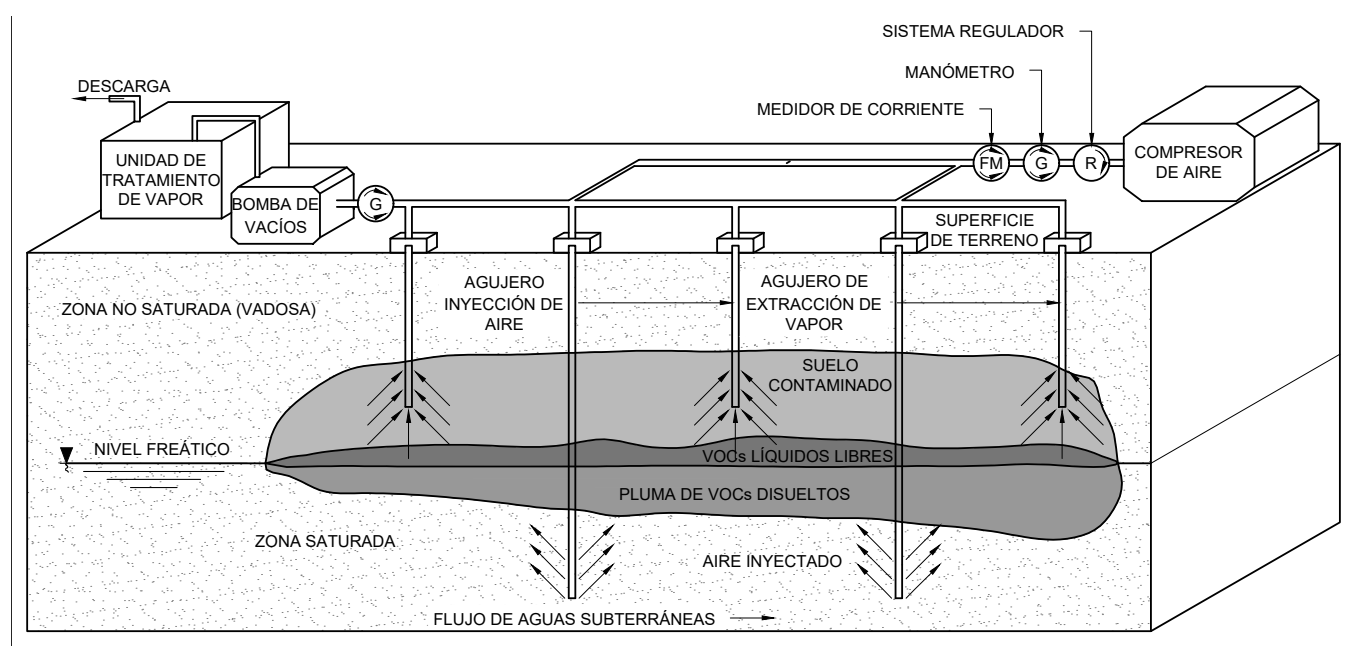

Figura 3. Esquema de sistema de volatilización de solventes

Fuente: modificado de (US EPA, 1992).

\section{Evalución de área desaturada y radio de influencia}

Para medir el radio de influencia de la inyección de aire, (Ishihara, Okamura y Oshita, 2003) ejecutaron una prueba centrífuga en un ensamblaje donde el aire se inyecta verticalmente a un modelo de suelo saturado, y encontraron que el diámetro de la región desaturada es de 5,0 m para una inyección de $95 \mathrm{kPa}$. (Tomida, 2014) realizó pruebas similares a un terraplén con una presión de inyección de $80 \mathrm{kPa}$ y descubre que el radio de desaturación aumenta con la presión y el tiempo de inyección, y se extiende a $9 \mathrm{~m}$ en 18 horas. Por otro lado, al comparar el método de desaturación con el método de pilas de arena compactada con distribución espacial típica menor a 2,5 m y presiones de inyección de $500 \mathrm{kPa}$, se reconoce que las pilas son conservadoras, ya que el suelo no solo se rigidiza sino que también es desaturado durante la inyección de compactación (Ishihara, Okamura y Oshita, 2003). Igualmente, el método de volatilización también se ha usado en suelos con presencia de contaminantes, y el trabajo de (Lundegard y LaBrecque, 1995) muestra métodos de medición del radio de influencia del suelo desaturado in situ. Se resalta que la medición precisa del radio de influencia permite un funcionamiento adecuado del sistema (Reddy y Adams, 2001). Un esquema típico del equipo de campo para volatilización de solventes se presenta en la Figura 3.

\section{Presión de inyección}

Según (Marulanda Culligan y Germaine, 2000), la presión de inyección debe ser mayor que la suma de la presión asociada a la cabeza de agua y las presiones capilares en el punto de inyección, y no debe exceder los esfuerzos efectivos en dicho punto para evitar fallas y agrietamiento del material. Experimentalmente, la presión de inyección a la que el suelo comienza a agrietarse equivale al $80 \%$ 
de los esfuerzos efectivos más la presión debida a la cabeza de agua en el punto de inyección (Okamura et al., 2011). (Yasuhara, Okamura y Kochi, 2008) encontraron que a mayor presión de inyección más amplia y uniforme es la desaturación del suelo, algo consistente con el trabajo de (Zeybek y Madabhushi, 2018). Por otro lado, (Zeybek y Madabhushi, 2017b) demostraron que la generación de un asentamiento excesivo de los suelos durante el proceso de inyección se da por presión de inyección excesiva que altera la estructura del suelo y causa grietas y fisuras, particularmente cerca del punto de inyección. Se reconoce que, aunque el aumento en la presión de inyección se considera una forma rápida y eficiente de desaturar y, en efecto, aumentar la resistencia a la licuefacción, esto debe abordarse en detalle, particularmente en el caso de la inyección bajo fundaciones superficiales.

(Ogata y Okamura, 2006) sugieren que la presión de inyección teórica máxima por encima de la cual el esqueleto del suelo se altera es $\left(P_{i n j}\right)_{\text {max }}=P_{h y d}+0,5 \sigma_{\nu}^{\prime}$, donde $\left(P_{i n j}\right)_{\text {max }}, P_{h y d}$ y $\sigma_{\nu}^{\prime}$ son la presión máxima de inyección, la presión hidrostática y el esfuerzo vertical efectivo en el punto de inyección, respectivamente. Además, (Zeybek y Madabhushi, 2017b) muestran que los asentamientos inducidos por la inyección son inevitables, independientemente de la presión de inyección. Sin embargo, según las observaciones, (Zeybek y Madabhushi, 2018) sugieren que la magnitud de los asentamientos puede reducirse significativamente si: a) se controla la respuesta de la fundación aumentando la presión gradualmente; b) la presión de inyección neta aplicada $\left(P_{i n j}\right)_{n e t}$, está dada por $\left(P_{i n j}\right)_{n e t}=\left(P_{i n j}\right)_{\max }-\left(P_{i n j}\right)_{\min }$ y es inferior al $10 \%$ de $\sigma_{\nu}^{\prime}$. Sin embargo, una presión de aire baja se convierte en un desafío para alcanzar áreas de desaturación uniformemente distribuidas y las burbujas de aire podrían tomar rutas de flujo preferenciales (Reddy, Kosgi y Zhou, 1995).

\section{Experimentación}

De acuerdo con los resultados obtenidos de ensayos realizados en mesa vibratoria (Camp, Camp y Andrus, 2010,Kohno y Nishigaki, 1982), centrífuga (Zeybek y Madabhushi, 2017b) y en campo (Okamura et al., 2011), es posible demostrar que el área de influencia y distribución del $S_{w}$ se ve afectada por el tipo de suelo, la estratificación, la presión y la profundidad de inyección (Yasuhara, Okamura y Kochi, 2008). Por otro lado, en el trabajo presentado por (Zeybek y Madabhushi, 2017a), se observa que el $S_{w}$ también se ve influenciado por la ocurrencia de cargas cíclicas, presentando un aumento de $0,64 \%$ y $0,41 \%$ para aceleraciones altas y bajas, respectivamente; información complementaria se encuentra en (Marasini y Okamura, 2015b). (Ishihara, Okamura y Oshita, 2003) desarrollaron pruebas de cortante cíclico no drenado en muestras con $S_{w}$ entre $68,8 \%$ y 92,6\%, indicando que la relación de esfuerzos para el suelo desaturado dobla la condición saturada. Resultados similares se pueden observar en (Okamura et al., 2009, Okamura, Ishihara y Tamura, 2006, Okamura, Ishihara y Tamura, 2003), y (Okamura et al., 2011). Particularmente, Okamura, (Ishihara, Okamura y Oshita, 2003) dejan claro que la resistencia al cortante cíclico de arena suelta puede mejorarse, pero el comportamiento de la deformación no cambia ya que la fase sólida permanece en una condición suelta. En otro orden de 
ideas, (Okamura et al., 2009) muestran que la resistencia a la licuefacción es proporcional a la succión matricial, siendo una relación creciente mayor que la del esfuerzo neto, y (Zeybek y Madabhushi, 2018) observan que la desaturación es menos consistente a profundidades con esfuerzos de confinamiento bajos y que la presencia de burbujas de aire minimizan la profundidad de licuefacción.

(Zeybek y Madabhushi, 2017a,Zeybek y Madabhushi, 2017b) estudiaron la deformación del suelo desaturado con y sin cimentaciones superficiales y después de cargas dinámicas, obteniendo una reducción del asentamiento postsísmico (reconsolidación) y de las deformaciones desviadoras al disminuir el grado de saturación; esto llevó al incremento de la capacidad de carga posterior a la sacudida, pero intensificando las aceleraciones transferidas a la fundación. Así mismo, (Marasini y Okamura, 2015a) analizaron un suelo con $S_{w}$ entre $100 \%$ y $85 \%$ portante de cimientos poco profundos, lo que resulta en que la desaturación limita el asentamiento de la fundación (un $70 \%$ y $50 \%$ en los modelos de carga liviana y pesada, respectivamente) y la generación de exceso de presión de poros (un $50 \%$ y $70 \%$ menos que el saturado), evitando la licuefacción. Igualmente, (Okamura y Teraoka, 2005a) establecen que después de la prueba cíclica, el asentamiento para el suelo desaturado equivale a aproximadamente la sexta parte del obtenido para el suelo saturado. Un análisis similar con y sin cimentación es presentado por (Takemura et al., 2009) y complementado por (Okamura y Teraoka, 2005a), lo que muestra que la inyección de aire tiene efectos significativos en la resistencia a la licuefacción para profundidades superiores a 2,0 m.

\section{Modelación numérica}

En algunos de los trabajos realizados para modelar la desaturación de suelos por el método de desaturación, se destaca la utilización del simulador de flujo multifásico TOUGH2 (Pruess, 1991), con el cual se ha logrado replicar la desaturación para diferentes presiones de inyección en el tiempo y espacio, obteniendo una concordancia entre mediciones experimentales y numéricas (Yasuhara, Okamura y Kochi, 2008). (Okamura et al., 2011) muestran que las predicciones de desaturación son coherentes con imágenes por tomografía y con muestras de laboratorio. Por otro lado, (Mitsuji, 2008) predice la respuesta dinámica con un análisis de esfuerzo efectivo, indicando que la velocidad, los desplazamientos y la deformación por cortante disminuyen debido a la desaturación. Así mismo, (Gao, Liu y Yu, 2013) estudian la evolución del exceso de presión de poros durante una desaturación variable. Estudios similares llevados a cabo por (Yashima, Oka, Taguchi y Tateishi, 1995) abordan el terremoto de Hyogoken Nambu de 1995 en Port Island (Kobe, Japón), calculando y replicando el exceso de presión de poros y la aceleración como respuestas a cargas dinámicas.

Por otro lado, (Marasini y Okamura, 2015b) utilizan el modelo LIQCA 2D propuesto por (Oka et al., 1999) y (Oka et al., 1994), donde se simula el módulo volumétrico del fluido para un $S_{w}=85 \%$, replicando el exceso de presiones de poros, el asentamiento estructural y deformación de los suelos de fundación observados en pruebas de centrífuga. (Takemura et al., 2009) muestran que la resisten- 
cia a la licuefacción para predicción y experimento, con $S_{w}=80 \%$, es aproximadamente 2,5 veces la de la saturación total. (McCray, 2000) presenta el estado del arte de los modelos matemáticos de volatilización de solventes, e indica que los resultados están ligados a una debida calibración; además, resalta que dichos modelos pueden adaptarse a las necesidades de la técnica de desaturación. Es de mencionar que la infiltración de aire, la desaturación y la deformación son procesos concurrentes que deben predecirse rigurosamente. En este sentido, trabajos como el de (Ramos, 2015), desde metodologías de estado crítico, o el de (Ramos, Prada y Vega, 2016), que evalúan esfuerzos y deformaciones en suelos granulares de manera rigurosa, son valiosos.

\section{MODELO MATEMÁTICO}

La formulación matemática presentada por (Pinder y Gray, 2008), que se basa en el trabajo realizado por (Darcy, 1983), se propone en este trabajo. Considerando el flujo bifásico en un medio poroso, las Ecuaciones (1) y (2) de balance para las fases no humectante, subíndice $i=n w$, y humectante, subíndice $i=w$, son como sigue:

$$
\begin{gathered}
\theta \frac{\partial S_{w}}{\partial t}+\nabla \cdot\left[-\frac{k_{i n t} k_{r, w}}{\mu_{w}}\left(\nabla p_{w}+\rho_{w} g \nabla D\right)\right]=0 \\
\theta \frac{\partial S_{n w}}{\partial t}+\nabla \cdot\left[-\frac{k_{i n t} k_{r, n w}}{\mu_{n w}}\left(\nabla p_{n w}+\rho_{n w} g \nabla D\right)\right]=0
\end{gathered}
$$

Donde: $\theta$ es la porosidad total; $S_{i}$ es el grado de saturación del fluido $i$ ( $w=$ agua, $n w=$ aire); $t$ es tiempo; $k_{i n t}$ es permeabilidad intrínseca del material $\left(m^{2}\right) ; k_{r, i}$ es la permeabilidad relativa asociada al fluido $i ; \mu_{i}$ es la viscosidad dinámica del fluido $i$ (Pa s); $p_{i}$ es la presión del fluido $i$ (Pa); $\rho_{i}$ es la densidad del fluido $i\left(\mathrm{~kg} / \mathrm{m}^{3}\right) ; g$ es la aceleración de la gravedad; y $D$ es la coordenada de la elevación vertical (m). La saturación de las fases se puede expresar mediante la Ecuación (3):

$$
S_{w}+S_{n w}=1
$$

La presión capilar a escala macro $\left(p_{c}\right)$ se puede definir como la Ecuación (4):

$$
p_{c}=p_{n w}-p_{w}
$$

Las expresiones anteriores simplifican las Ecuaciones (1) y (2), eliminando dos incógnitas, y la ecuación de conservación de masa del fluido en los poros solo depende de $p_{n w}$ y $S_{n w}$. Dado que la presión capilar se rige por $S_{w}$, el término capilar, $\nabla p_{c}$, se puede expresar como la Ecuación (5):

$$
\nabla p_{c}=\frac{\partial p_{c}}{\partial S_{w}} \nabla S_{w}
$$


Sustituyendo las Ecuaciones (3), (4) y (5) en la Ecuación (1) y agregando la Ecuación (2), el sistema de conservación de la masa se escribe en forma de presión saturación y se expresa en las Ecuaciones (6) y (7), respectivamente, como:

$$
\begin{gathered}
{\left[-\frac{k_{i n t} k_{r, w}}{\mu_{w}}\left(\nabla p_{n w}+\rho_{w} g \nabla D-\frac{\partial p_{c}}{\partial S_{w}} \nabla S_{w}\right)-\frac{k_{i n t} k_{r, n w}}{\mu_{n w}}\left(\nabla p_{n w}+\rho_{n w} g \nabla D\right)\right]=0} \\
\theta \frac{\partial S_{n w}}{\partial t}+\nabla \cdot\left[-\frac{k_{i n t} k_{r, n w}}{\mu_{n w}}\left(\nabla p_{n w}+\rho_{n w} g \nabla D\right)\right]=0
\end{gathered}
$$

A partir de las propiedades hidráulicas de los suelos no saturados, el grado efectivo de saturación de la fase w viene dado por la Ecuación (8):

$$
S_{w, e f f}=\left(\frac{S_{w}-S_{\min }}{S_{\max }-S_{\min }}\right)
$$

Donde: $S_{\max }$ y $S_{\min }$ son los valores límite máximos y mínimos de la fase líquida $\left(S_{w}\right)$, respectivamente. La permeabilidad relativa de las fases de agua y aire viene dada por las Ecuaciones (9) y (10), respectivamente:

$$
\begin{aligned}
k_{r, w} & =S_{w, e f f}^{a}\left[1-\left(1-\left(S_{w, e f f}\right)^{1 / m}\right)^{m}\right]^{2} \\
k_{r, n w} & =\left(1-S_{w, r e f f}\right)^{b}\left(1-\left(S_{w . e f f}\right)^{1 / m}\right)^{2 m}
\end{aligned}
$$

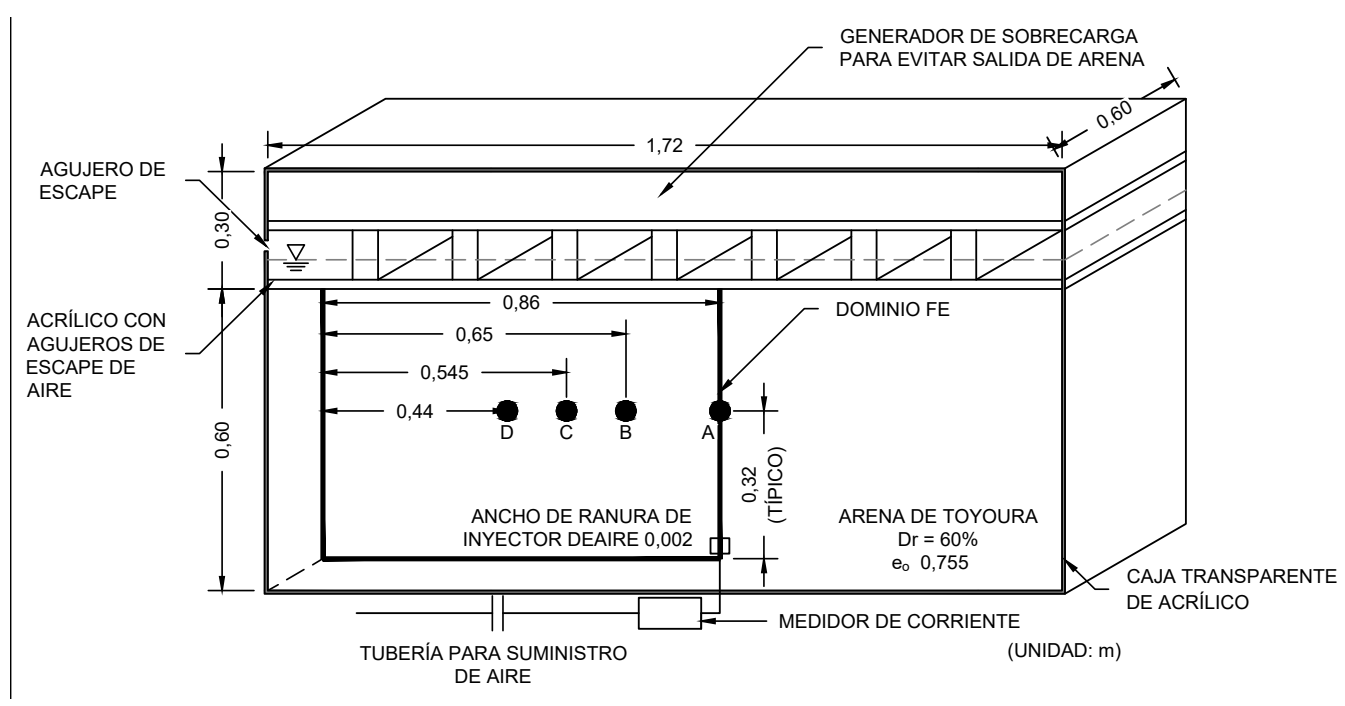

Figura 4. Esquema caja acrílica transparente. Los valores de $S_{w}$ se miden en los lugares A, B, C y D

Fuente: modificado de (Yasuhara, Okamura y Kochi, 2008) 
Donde: $a$ y $b$ son los parámetros de forma y $m$ un parámetro de material. Ahora, la expresión para la curva característica suelo y agua viene dada por la Ecuación (11) (Van Genuchten, 1980):

$$
p_{c}=\frac{1}{\alpha}\left(S_{w, e f f}^{-1 / m}-1\right)^{1-m}
$$

Donde: $\mathrm{n}$ es un parámetro de material que cumple la expresión $m=1-(1 / n)$, y $\alpha$ es el inverso del valor de entrada de aire, el cual forma parte del modelo de (Van Genuchten, 1980). Derivando la Ecuación (11), el término capilar en la Ecuación (6) se expresa como la Ecuación (12) (equivalente al trabajo de (Horgue et al., 2015)):

$$
\frac{\partial p_{c}}{\partial S_{w}}=-\frac{1-m}{\alpha m}\left(\left(S_{w, e f f}\right)^{-1 / m}-1\right)^{-m}\left(S_{w, e f f}\right)^{-\frac{1+m}{m}}
$$

\section{ANÁLISIS Y MODELACIÓN DE INYECCIÓN DE GAS EN MATERIAL SATURADO}

\section{Validación del modelo propuesto}

(Yasuhara, Okamura y Kochi, 2008) desaturaron por inyección de aire una columna de suelo completamente saturada, y realizaron la validación por medio de un análisis numérico. Las condiciones experimentales fueron: flujo de agua y aire en la parte superior de la caja, presión hidrostática inicial para fase húmeda, y flujo de aire a través de un inyector en la parte central inferior de la caja. La Figura 4 muestra un esquema del montaje utilizado por los autores.

Los resultados de (Yasuhara, Okamura y Kochi, 2008) se utilizan para validar la formulación matemática y el análisis numérico propuesto en el presente trabajo. Los parámetros del suelo, las presiones de inyección y las condiciones iniciales y de frontera utilizadas por (Yasuhara, Okamura y Kochi, 2008) son presentados en la tabla I y en la Figura 4, respectivamente.

La Figura 5 muestra la comparación de los resultados experimentales y numéricos de (Yasuhara, Okamura y Kochi, 2008) con el análisis numérico de desaturación propuesto en este trabajo. La Figura 5a ilustra discrepancias entre los resultados experimentales en (Yasuhara, Okamura y Kochi, 2008) y las predicciones con el enfoque propuesto en este trabajo. Estas discrepancias se atribuyen directamente a inconsistencias experimentales y problemas de calibración de sensores que se obtuvieron en el experimento de (Yasuhara, Okamura y Kochi, 2008) y en menor medida podrían también deberse a irregularidades entre permeabilidades reales y medidas, y la curva característica.

De igual manera, (Yasuhara, Okamura y Kochi, 2008) al respecto de sus resultados, expresan que los flujos predichos subestiman las mediciones experimentales, sin embargo, cuanto mayor es la presión de inyección, menor es la discrepancia. Además, las predicciones indican que el aire llega a la ubicación D, lo cual no es congruente con las mediciones experimentales. Sin embargo, para otras 
ubicaciones A, B y C, y para tiempos mayores, las predicciones se acercan a las reales a medida que aumentan las presiones de aire. Por otro lado, se piensa de igual manera que las características de drenaje del suelo pudieron no replicarse teóricamente, por circunstancias asociadas a los experimentos de retención de agua. En otras palabras, (Yasuhara, Okamura y Kochi, 2008) mencionan que las propiedades como permeabilidad intrínseca y relativa, y características de drenaje del suelo deben examinarse con rigurosidad, antes de realizar predicciones del fenómeno.

La Figura 5b muestra un comparativo entre las predicciones de (Yasuhara, Okamura y Kochi, 2008) y las predicciones con el enfoque propuesto en este trabajo. Se resalta que la Figura 5 se presenta hasta los $6000 \mathrm{~s}$, esto se debe a que los experimentos planteados por (Yasuhara, Okamura y Kochi, 2008) se efectuaron hasta dicho periodo. Además, el grado de saturación residual al final del experimento es de aproximadamente el $86 \%$.

Por otro lado, para las predicciones del presente estudio se percibe que para los puntos B, C y D se alcanza un grado de saturación a los 6000 s similar al del estudio de (Yasuhara, Okamura y Kochi, 2008), pero para el punto D se nota que en el proceso de saturación hay un grado se saturación a los 6000 s menor que el determinado en el experimento de (Yasuhara, Okamura y Kochi, 2008). Sin embargo, al final de la predicción se percibe que la cantidad de aire atrapado, después de que se detiene la inyección de aire, es independiente de la presión de inyección y está estrechamente ligado al campo gravitacional. Esta Figura muestra una similitud entre los resultados obtenidos por las dos metodologías. Sin embargo, se observa un retraso de tiempo del $S_{w}$ en A, B, C y D, de los resultados

Tabla I. Parámetros de material e hidráulicos utilizados para predicciones

\begin{tabular}{ccc}
\hline Variable & Magnitud & Descripción \\
\hline$\rho_{w}$ & $1000 \mathrm{~kg} / \mathrm{m}^{3}$ & Densidad de fluido, fase húmeda \\
\hline$\mu_{w}$ & $1 \times 10^{-3} \mathrm{~Pa} \mathrm{~s}$ & Viscosidad dinámica, fase húmeda \\
\hline$\rho_{n w}$ & $1,28 \mathrm{~kg} / \mathrm{m}^{3}$ & Densidad de fluido, fase no húmeda \\
\hline$\mu_{n w}$ & $1,81 \times 10^{-5} \mathrm{~Pa} \mathrm{~s}$ & Viscosidad dinámica, fase no húmeda \\
\hline$\theta$ & 0,421 & Porosidad \\
\hline$S_{m a x}$ & 1,0 & Máximo grado de saturación, fase de húmeda \\
\hline$\alpha$ & $2,237 \times 10^{-4} 1 / \mathrm{Pa}$ & Parámetro $\alpha$ de Van Genuchten \\
\hline$n$ & 8,696 & Parámetro material $n$ Van Genuchten \\
\hline$a$ & 0,50 & Parámetro de forma, fase húmeda \\
\hline$b$ & 0,33 & Parámetro de forma, fase no húmeda \\
\hline$k_{i n t}$ & $2,04 \times 10^{-11} \mathrm{~m}^{2}$ & Permeabilidad intrínseca \\
\hline
\end{tabular}

Fuente: elaboración propia. 

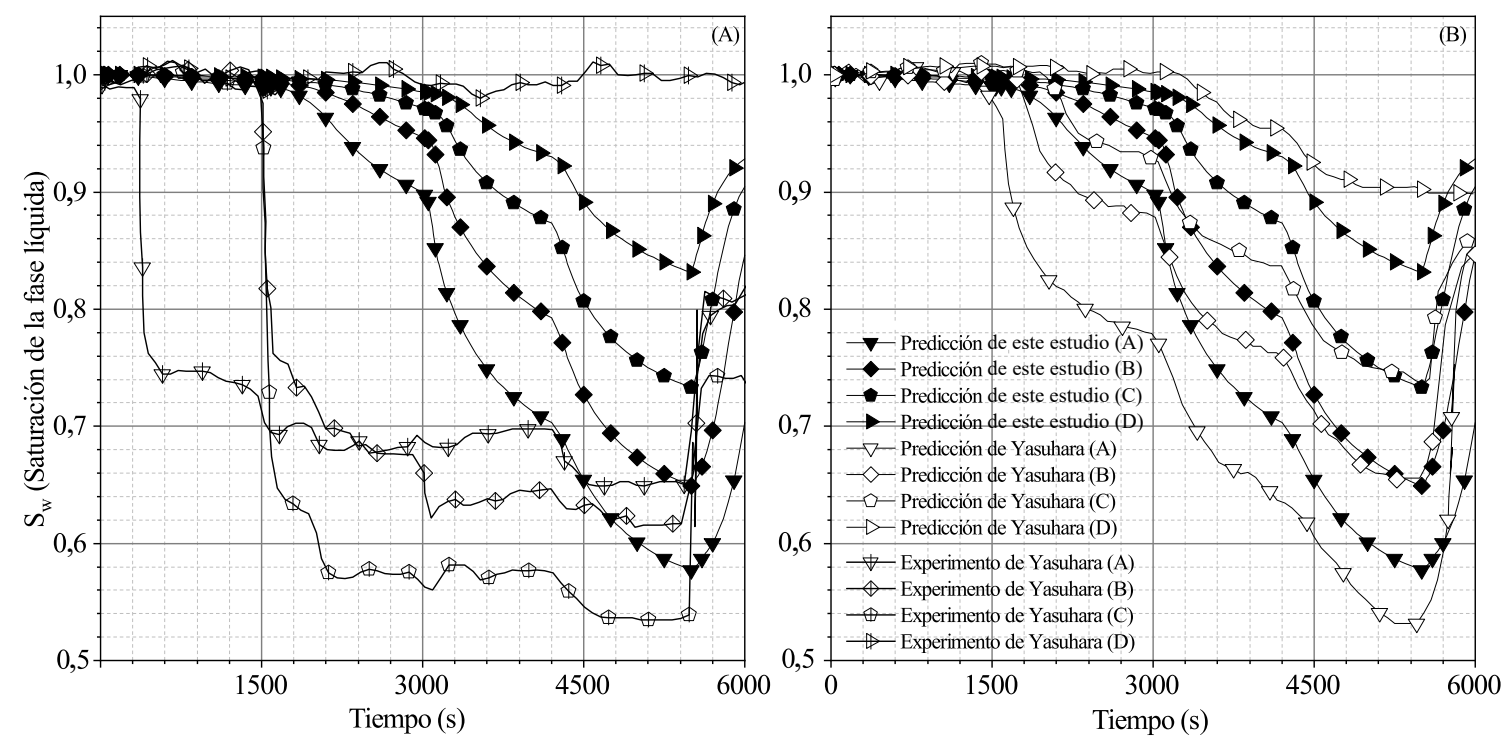

Figura 5. Comparación entre el presente trabajo y predicciones de (Yasuhara, Okamura y Kochi, 2008) para $S_{w}$

Fuente: elaboración propia.

de (Yasuhara, Okamura y Kochi, 2008), explicado por las diferencias entre las metodologías de los modelos numéricos y las funciones de permeabilidad relativa.

Al comparar ambas simulaciones numéricas se observan las siguientes tendencias: 1) $S_{w}$ inicialmente disminuye cerca del inyector y progresivamente alrededor, debido a las fuerzas boyantes y los gradientes inducidos; 2) hay tendencia similar en desaturación, así como un valor mínimo equivalente de $S_{w}$ al final de la inyección en $\mathrm{t}=5500 \mathrm{~s}$, y 3) después de $\mathrm{t}=5500 \mathrm{~s}$, el $S_{w}$ aumenta debido al apagado del inyector; no obstante, el aire permanece en el dominio, implicando valores de $S_{w}$ cercanos a 0,9 en $\mathrm{t}=6000 \mathrm{~s}$. Por tanto, a partir de las simulaciones, se observa que el flujo bifásico implementado en el modelo de elementos finitos propuesto captura las tendencias generales de la predicción y del experimento mostradas por (Yasuhara, Okamura y Kochi, 2008).

\section{Análisis paramétrico de inyección de aire}

Se realiza un estudio paramétrico para evaluar la influencia de la permeabilidad intrínseca y las presiones de inyección en el avance de aire dentro del suelo. Se seleccionaron tres tipos de suelo, cuyos parámetros hidráulicos se presentan en la tabla II, obtenidos de (Lu, y Likos, 2004). Estos parámetros son considerados representativos de arenas y arenas limosas, siendo sometidos a presiones de inyección de aire que varían de 75 a $85 \mathrm{kPa}$. En el presente análisis, la presión de inyección máxima de modo que el esqueleto del suelo se altere poco durante la inyección de aire, es equivalente a 87 $\mathrm{kPa}$ (para un inyector ubicado a 6,0 m bajo la superficie y un suelo con $\gamma=19,0 \mathrm{kN} / \mathrm{m}^{3}$ ) según lo sugerido por (Ogata y Okamura, 2006). 


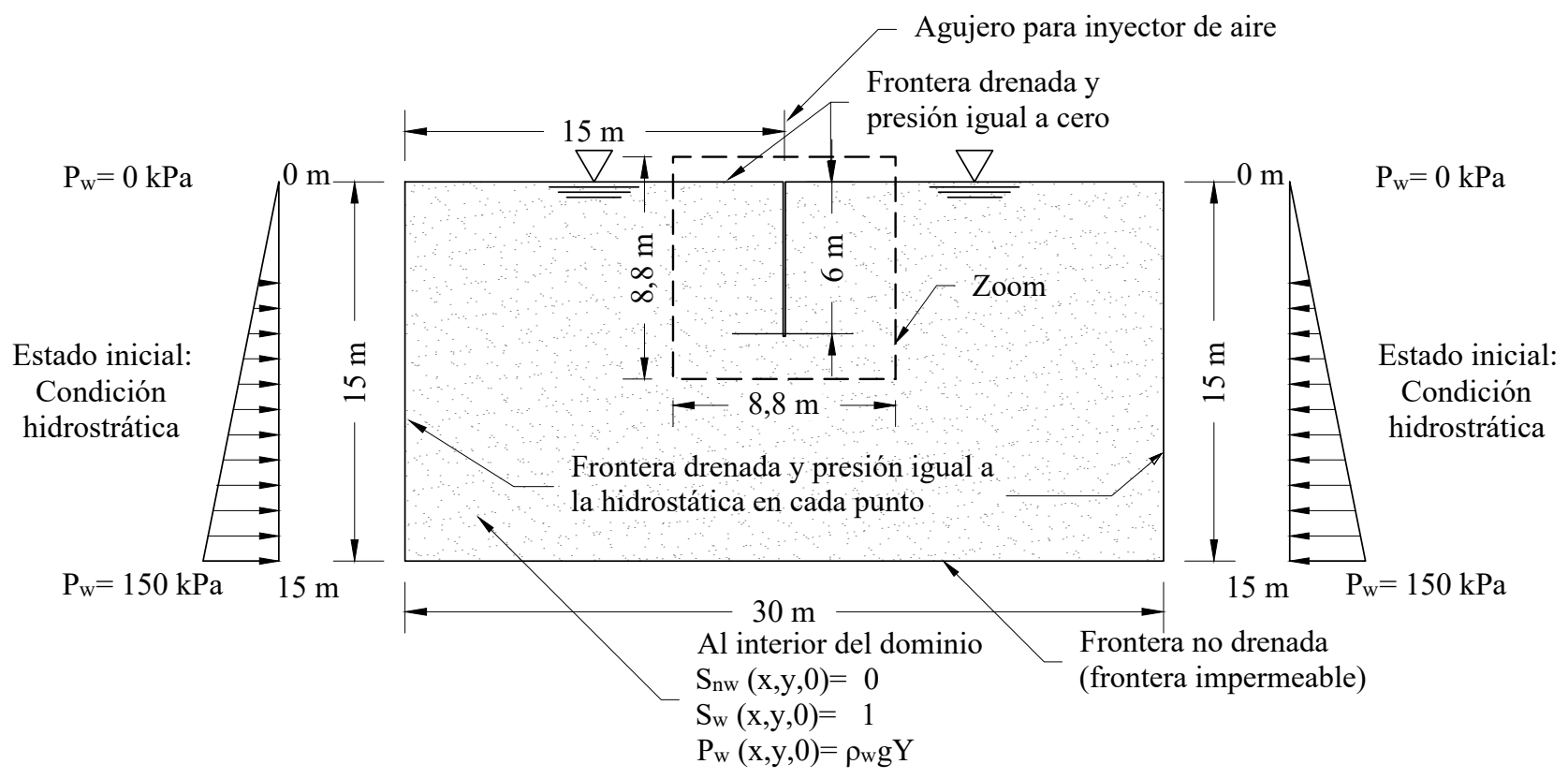

Figura 6. Modelo usado para el análisis paramétrico

Fuente: elaboración propia.

El modelo utilizado consiste en un suelo homogéneo con dimensiones de $30 \mathrm{~m}$ de ancho y 15 $\mathrm{m}$ de profundidad (Figura 6). Para la fase gaseosa, se asigna una condición de impermeabilidad al fondo, y se permite flujo de gas en el resto de fronteras, así como en el punto donde se encuentra el inyector a 6,0 $\mathrm{m}$ de profundidad. Las simulaciones inician desde una condición saturada y la fase gaseosa fluye cuando la presión generada en el inyector supera la presión hidrostática.

Tabla II. Parámetros utilizados para el grupo de simulaciones

\begin{tabular}{cccc}
\hline Suelo & $\alpha(1 / k P a)$ & $n$ & $k_{\text {int }}\left(m^{2}\right)$ \\
\hline 1 & 0,07 & 2,0 & $5,55 \times 10^{-12}$ \\
\hline 2 & 0,10 & 4,0 & $1,00 \times 10^{-11}$ \\
\hline 3 & 0,12 & 6,0 & $5,55 \times 10^{-11}$
\end{tabular}

Nota: $a=3,0 ; b=0,33 \overline{; \rho_{w}}=1000 \mathrm{~kg} / \mathrm{m}^{3} ; \mu_{w}=1 \times 10^{-3} \mathrm{~Pa}, \rho_{n w}=1,28 \mathrm{~kg} / \mathrm{m}^{3}, \mu_{n w}=$

$$
1,81 \times 10^{-5} \mathrm{~Pa}, \theta=0,34, S_{w, \max }=1,0 \text { y } S_{w, \min }=0,0 .
$$

Datos de (Chen, Hopmans y Grismer, 1999).

Fuente: elaboración propia. 


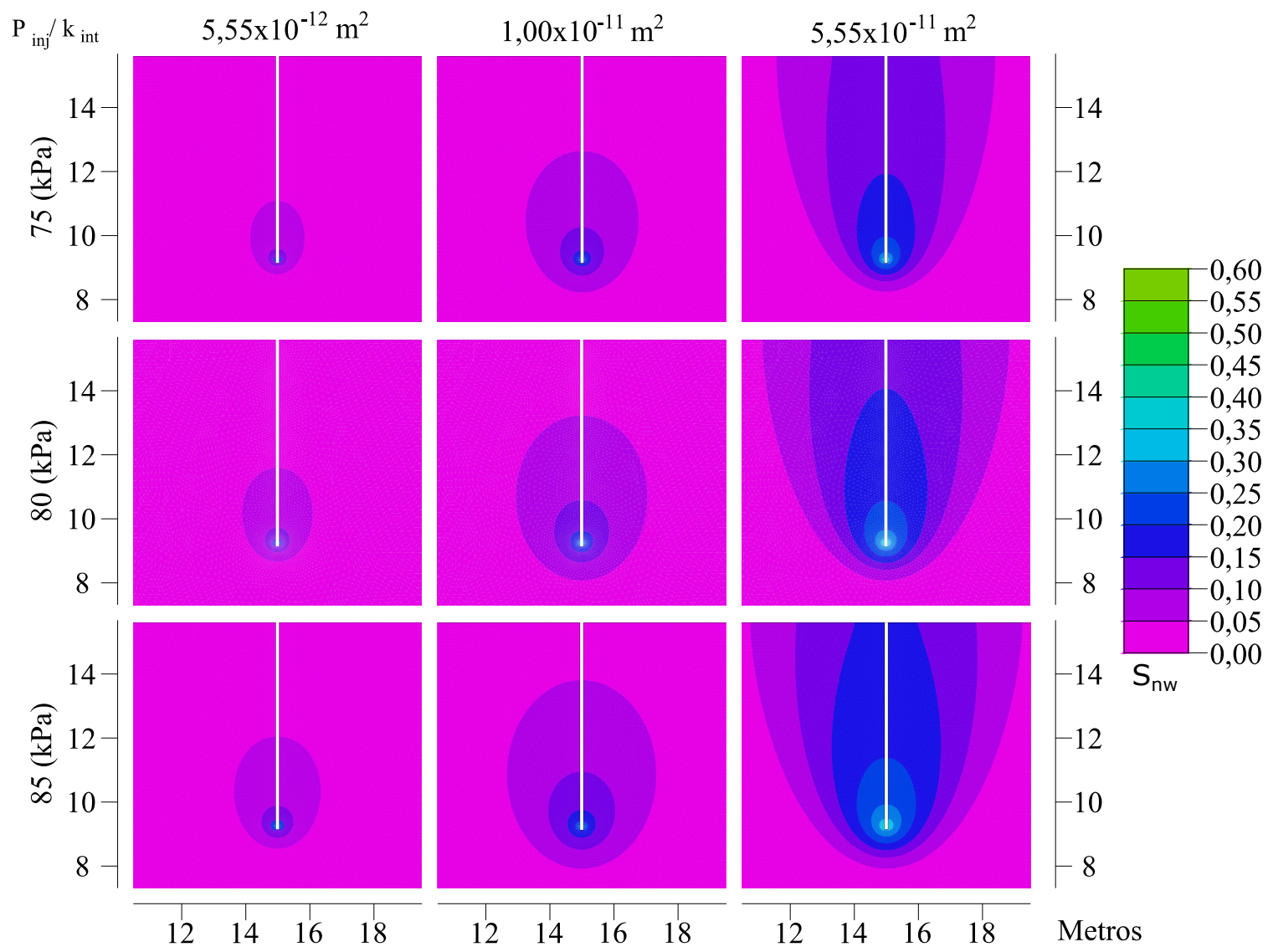

Figura 7. Isocontorno de $S_{n w}$ en función de $P_{n w, i n j}$ para t $=1800$ s. Distancia en metros

Fuente: elaboración propia.

La Figura 7 presenta los isocontornos de la evolución de $S_{n w}$ en $\mathrm{t}=1800 \mathrm{~s}$ como función de la presión de inyección y la permeabilidad intrínseca. Se aclara que los isocontornos corresponden a un zoom fijo con forma cuadrada de 8,8 m de lado, ilustrado con líneas discontinuas en la Figura 6. El borde inferior izquierdo del zoom se encuentra en las coordenadas $x=10,6 \mathrm{~m}$ y $y=7,2 \mathrm{~m}$. En la misma Figura se observa que, para una presión de inyección fija, cuanto más permeable es el suelo, mayor es la infiltración de la fase gaseosa. En consecuencia, para permeabilidades fijas e incrementos de presión de inyección de aire, se observa un aumento del avance del frente de desaturación, siendo en todos los casos, predominantemente vertical debido a las fuerzas gravitacionales.

Por otro lado, la predicción del impacto de la permeabilidad y la presión de inyección en el flujo de la fase gaseosa y el radio efectivo se muestra en la Figura 8. Se selecciona el valor de $S_{n w}=0,1$ porque, como lo muestran (Chaney, 1978) y (Yoshimi, Tanaka y Tokimatsu, 1989), la susceptibilidad a la licuefacción de arenas completamente saturadas disminuye a la mitad cuando el grado de saturación del suelo se reduce en $10 \%$. Debido a que el aire inyectado penetra y genera un radio efectivo desde el inyector, este definirá el patrón (triangular o cuadrado) y el espaciado de las perforaciones 

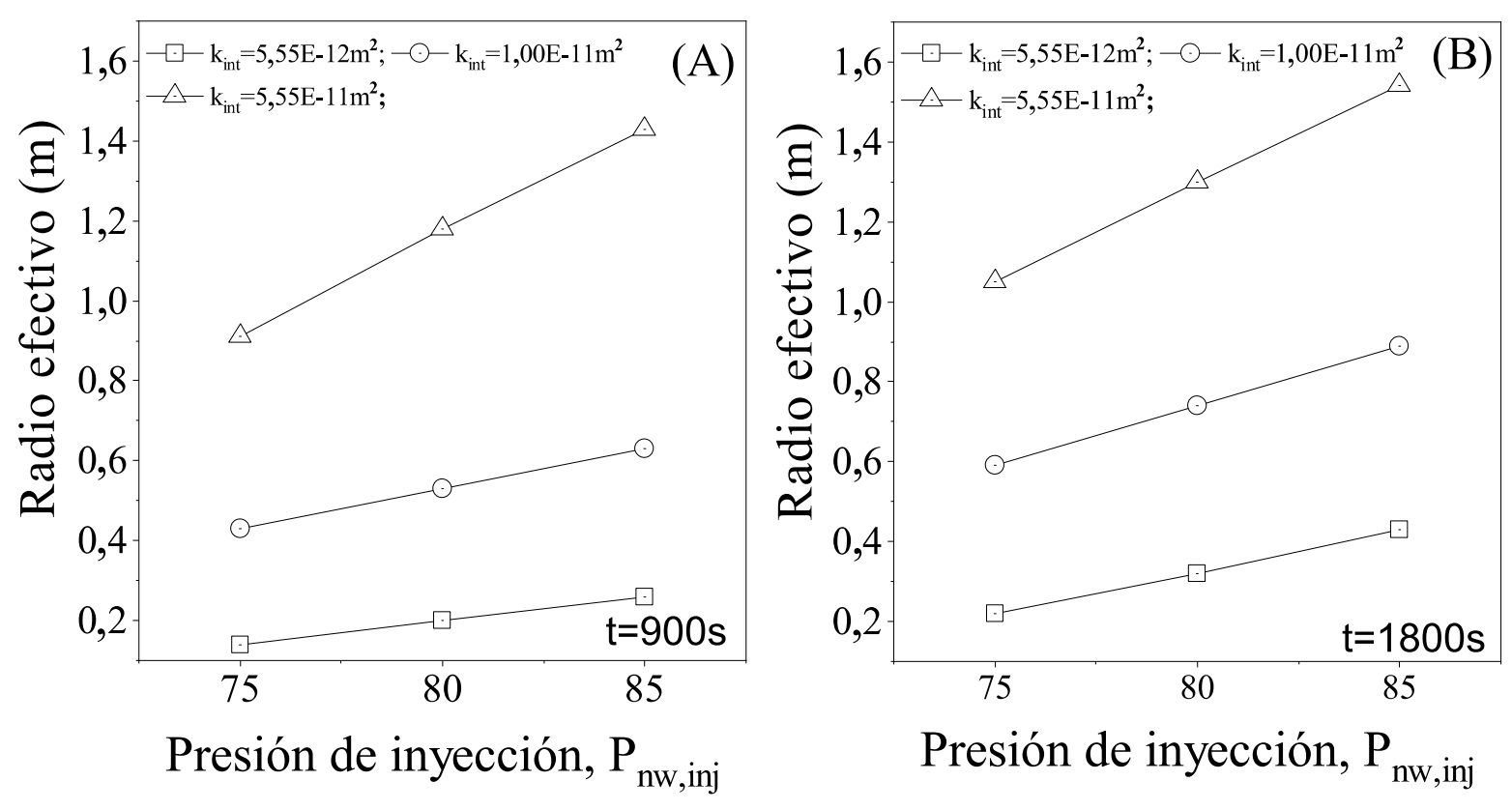

Figura 8. Radio efectivo para los suelos en la tabla II $S_{n w}=0,1$ y $P_{n w, i n j}$ de 70 a $85 \mathrm{kPa}$

Fuente: elaboración propia.

de inyección de aire que deben diseñarse en función de los requisitos del proyecto y la cobertura completa del área susceptible a licuefacción dentro de la profundidad a mejorar.

Las lecturas de las magnitudes del radio de desaturación de las Figuras 8A y 8B se tomaron en tiempos iguales a 900 s y 1800 s, respectivamente. Dichas Figuras presentan los valores de radio efectivo para los suelos de la tabla II, lo que indica que para todas las presiones el radio efectivo aumenta con el tiempo y el efecto es mayor a presiones de inyección más altas. Las Figuras también muestran que, para una misma presión de inyección, se observa un incremento del radio efectivo de desaturación a medida que se incrementa la permeabilidad intrínseca del suelo. Asimismo, cuando se comparan las Figuras 8A y 8B se ven resultados para iguales presiones más cercanos en el suelo menos permeable y más distantes en el suelo más permeable. En dichas Figuras se observa que para valores bajos de permeabilidades intrínsecas, el radio efectivo crece lentamente con incrementos en la presión de inyección; en cambio, para el suelo más permeable, el aumento en el radio efectivo es más pronunciado.

\section{CONCLUSIONES}

La inducción parcial del grado de saturación en suelos para la mitigación de la licuefacción sigue siendo una tecnología relativamente nueva que necesita ser estudiada. Se debe profundizar en la comprensión de los mecanismos del flujo en medios porosos, en busca de mejorar su implementación 
en casos reales, ya que la mayor parte del trabajo de laboratorio realizado hasta el día de hoy incluye simplificaciones que pueden restringir la aplicabilidad de los resultados. De igual forma, como consecuencia de los pocos sistemas diseñados, operados y monitoreados en campo, no existen datos suficientes de rendimiento que permitirían reconocer con mayor certeza las ventajas y limitaciones de la técnica.

Por esto, es fundamental investigar sobre el control más preciso del grado de saturación generado en los suelos por procesos de inyección de aire, así como su distribución y extensión, la longevidad del efecto de desaturación y su rentabilidad respecto al área de ejecución.

Como resultado de este trabajo, se ha establecido una implementación para la predicción del flujo bifásico en el suelo a través de los criterios (estándares) de un flujo bifásico isotérmico, incompresible e inmiscible con impactos de succión matricial. Los resultados numéricos del modelo están en concordancia con los obtenidos por (Yasuhara, Okamura y Kochi, 2008). Por otro lado, se muestran discrepancias significativas con respecto a las mediciones experimentales desarrolladas por los mismos autores.

De los modelos realizados se puede concluir que el grado de saturación de la fase húmeda disminuye, sobre todo, cerca del inyector y progresivamente hacia arriba, representando el avance del frente de gas. De igual forma, a medida que la presión de inyección de gas es mayor, el grado de saturación de la fase húmeda disminuye, lo que permite gradientes más altos para el flujo de gas. Por otra parte, se encontró que, para suelos con baja permeabilidad, el radio efectivo es relativamente pequeño para presiones bajas y altas de inyección; por el contrario, cuando la permeabilidad es mayor, se obtienen mayores variaciones del radio efectivo en el mismo instante de tiempo, causado principalmente por las fuerzas boyantes y los gradientes inducidos.

Por último, la magnitud de la extensión del frente de la fase gaseosa durante y después del proceso de inyección de aire está fuertemente controlada por la permeabilidad intrínseca y la presión de inyección, como lo indica el análisis paramétrico efectuado.

\section{REFERENCIAS}

[Bouferra, Benseddiq y Shahrour, 2007] Bouferra, R., Benseddiq, N. y Shahrour, I. (2007). Saturation and preloading effects on the cyclic behavior of sand. International Journal of Geomechanics, 7(5), 396-401. DOI: https://doi.org/10.1061/(ASCE) 1532-3641 (2007) 7:5 (396) 个Ver página 143

[Camp, Camp y Andrus, 2010] Camp, W. M., Camp, H. C. y Andrus, R. D. (2010). Liquefaction mitigation using air injection. En International Conferences on Recent Advances in Geotechnical Earthquake Engineering and Soil Dynamics. Missouri University of Science and Technology. $\uparrow$ Ver página 147 
[Chaney, 1978] Chaney, R. C. (1978). Saturation effects on the cyclic strength of sands. En Volume I of Earthquake Engineering and Soil Dynamics-Proceedings of the ASCE Geotechnical Engineering Division Specialty Conference (pp. 342-358). Junio 19-21. Pasadena, California: Geotechnical Engineering Division of ASCE. $\uparrow$ Ver página 155

[Chen, Hopmans y Grismer, 1999] Chen, J., Hopmans, J. y Grismer, M. (1999). Parameter estimation of two-fluid capillary pressure-saturation and permeability functions. Advances in Water Resources, 22(5), 479-493. DOI: https: / / doi .org/10.1016/s0309-1708 (98) 00025-6 个Ver página 154

[Darcy, 1983] Darcy, H. (1983). Determination of the laws of flow of water through sand. En R. A. Freeze y W. Back (eds.), Physical hydrogeology. Benchmark Papers in Geology (vol. 72). Stroudsburg: Hutchinson Ross. $\uparrow$ Ver página 149

[Eseller-bayat, Yegian y Alshawabkeh, 2013] Eseller-bayat, E., Yegian, M. K. y Alshawabkeh, A. (2013). Liquefaction response of partially saturated sands. I : experimental results. Journal of Geotechnical and Geoenvironmental Engineering, 139(6), https: / / doi . org/10 . 1061/(ASCE) GT . 1943-5606.0000815. $\uparrow$ Ver página 142, 145

[Gallagher, Pamuk y Abdoun, 2007] Gallagher, P. M., Pamuk, A. y Abdoun, T. (2007). Stabilization of liquefiable soils using colloidal silica grout. Journal of Materials in Civil Engineering, 19(1), 33-40. DOI: https://doi.org/10.1061/(ASCE) 0899-1561(2007) 19:1(33) 个Ver página 142

[Gao, Liu y Yu, 2013] Gao, Q., Liu, Z. y Yu, X. (2013). Computer simulations on the effects of desaturation on soil liquefaction resistance. En IACGE, 786-795. Reston, EE. UU.: American Society of Civil Engineers. DOI: https://doi.org/10.1061/9780784413128.091 $\uparrow$ Ver página 148

[Grozic, Nadim y Kvalstad, 2005] Grozic, J. L. H., Nadim, F. y Kvalstad, T. J. (2005). On the undrained shear strength of gassy clays. Computers and Geotechnics, 32(7), 483-490. DOI: https : / / doi . org/ $10.1016 / j$.compgeo.2005.10.002 个Ver página 143

[Grozic, Robertson y Morgenstern, 1999] Grozic, J. L., Robertson, P. K. y Morgenstern, N. R. (1999). The behavior of loose gassy sand. Canadian Geotechnical Journal, 36(3), 482-492. DOI: https: // doi.org/10.1139/t99-007个Ver página 143

[He, Chu e Ivanov, 2013] He, J., Chu, J. e Ivanov, V. (2013). Mitigation of liquefaction of saturated sand using biogas. Géotechnique, 63(4), 267-275. DOI: https://doi .org/10.1680/geot. SIP13.P.004 个Ver página 142

[Holbrook et al., 1998] Holbrook, T. B., Bass, D., Boersma, P., DiGiulio, D. C., Eisenbeis, J., Hutzler, N. J. y Roberts E. (1998). Vapor extraction and air sparging design and application. wastech innovative site remediation technology series (vol. 7). Annapolis, EE. UU. $\uparrow$ Ver página 145 
[Horgue et al., 2015] Horgue, P., Soulaine, C., Franc, J., Guibert, R. y Debenest, G. (2015). An opensource toolbox for multiphase flow in porous media. Computer Physics Communications, 187, 217226. DOI: https://doi.org/10.1016/j.cpc.2014.10.005个Ver página 151

[Ishihara, Huang y Tsuchiya, 1998] Ishihara, K., Huang, Y. y Tsuchiya, H. (1998). Liquefaction resistance of nearly saturated sand as correlated with longitudinal wave velocity. En J. F. Thimus et al. (eds.), Poromechanics: a tribute to Maurice A. Biot (pp. 583-586). Boca Ratón: CRC Press. https://doi.org/10.1201/9781003078487-98 ^Ver página 143

[Ishihara, Okamura y Oshita, 2003] Ishihara, M., Okamura, M. y Oshita, T. (2003). Desaturating sand deposit by air injection for reducing liquefaction potential. En Proc. Pacific Conference on Earthquake Engineering. Tsukuba City, Japón. $\uparrow$ Ver página 142, 146, 147

[Kohno y Nishigaki, 1982] Kohno, I. y Nishigaki, M. (1982). Some aspects of laboratory permeability test. Soils and Foundations, 22(4), 181-190. DOI: https: / / doi .org/10.3208/sandf1972.22. 4_181 $\uparrow$ Ver página 147

[Lu, y Likos, 2004] Lu, N. y Likos, W. J. (2004). Unsaturated soil mechanics. Hoboken, EE. UU.: Wiley. Recuperado de https://books.google.com.co/books?id=Rv1RAAAAMAAJ 个Ver página 153

[Lundegard y LaBrecque, 1995] Lundegard, P. D. y LaBrecque, D. (1995). Air sparging in a sandy aquifer (Florence, Oregon, U.S.A.): actual and apparent radius of influence. Journal of Contaminant Hydrology, 19(1), 1-27. DOI: https://doi .org/10.1016/0169-7722(95)00010-S 个Ver página 146

[Marasini y Okamura, 2015a] Marasini, N. P. y Okamura, M. (2015a). Air injection to mitigate liquefaction under light structures. International Journal of Physical Modelling in Geotechnics, 15(3), 129140. DOI: https://doi.org/10.1680/jphmg.14.00005 个er página 142, 148

[Marasini y Okamura, 2015b] Marasini, N. P. y Okamura, M. (2015b). Numerical simulation of centrifuge tests to evaluate the performance of desaturation by air injection on liquefiable foundation soil of light structures. Soils and Foundations, 55(6), 1388-1399. DOI: https : / / doi . org/ 10 . $1016 / j$.sandf.2015.10.005 个Ver página 147,148

[Marulanda Culligan y Germaine, 2000] Marulanda, C., Culligan, P. J. y Germaine, J. T. (2000). Centrifuge modeling of air sparging - a study of air flow through saturated porous media. Journal of Hazardous Materials, 72(2-3), 179-215. DOI: https: // doi .org/10.1016/s0304-3894 (99) $00140-5 \uparrow$ Ver página 146

[McCray, 2000] McCray, J. E. (2000). Mathematical modeling of air sparging for subsurface remediation: state of the art. Journal of Hazardous Materials, 72(2-3), 237-263. DOI: https : / / doi . org / 10 . 1016/S0304-3894(99)00142-9个Ver página 149 
[Mitsuji, 2008] Mitsuji, K. (2008). Numerical simulations for development of liquefaction countermeasures by use of partially saturated sand. En Proceedings of the 14th World Conference on Earthquake Engineering October (pp. 12-17). 个Ver página 148

[Ogata y Okamura, 2006] Ogata, H. y Okamura, M. (2006). Experimental study on air behaviour in saturated soil under air injection. En Proc. Symp. On Natural Disaster Prevention, JSCE (pp. 89-90). Tokushima, Japón. $\uparrow$ Ver página 147, 153

[Oka et al., 1994] Oka, F., Yashima, A., Shibata, T., Kato, M. y Uzuoka, R. (1994). FEM-FDM coupled liquefaction analysis of a porous soil using an elasto-plastic model. Applied Scientific Research, 52(3), 209-245. DOI: https://doi.org/10.1007/BF00853951 个Ver página 148

[Oka et al., 1999] Oka, F, Yashima, A., Tateishi, A., Taguchi, Y. y Yamashita, A. (1999). A cyclic elastoplastic constitutive model for sand considering a plastic-strain dependence of the shear modulus. Géotechnique, 49(5), 661-680. DOI: https://doi.org/10.1680/geot.1999.49.5.661 个Ver página 148

[Okamura y Noguchi, 2009] Okamura, M. y Noguchi, K. (2009). Liquefaction resistances of unsaturated non-plastic silt. Soils and Foundations, 49(2), 221-229. DOI: https: / / doi . org/10.3208/ sandf. $49.221 \uparrow$ Ver página 145, 147, 148

[Okamura y Soga, 2006] Okamura, M. y Soga, Y. (2006). Effects of pore fluid compressibility on liquefaction resistance of partially saturated sand. Soils and Foundations, 46(5), 695-700. DOI: https://doi.org/10.3208/sandf.46.695 个Ver página 142

[Okamura y Tamura, 2004] Okamura, M. y Tamura, K. (2004). Prediction method for liquefactioninduced settlement of embankment with remedial measure by deep mixing method. Soils and Foundations, 44(4), 53-65. DOI: https: / / doi .org/10.3208/sandf.44.4_53 个er página 145

[Okamura y Teraoka, 2005a] Okamura, M. y Teraoka, T. (2005a). Shaking table tests to investigate soil desaturation as a liquefaction countermeasure. En Seismic Performance and simulation of pile foundations in liquefied and laterally spreading ground (pp. 282-293). Reston, EE. UU.: American Society of Civil Engineers. DOI: https://doi.org/10.1061/40822 (184)23 个Ver página 145, 148

[Okamura y Teraoka, 2005b] Okamura, M. y Teraoka, T. (2005b). Shaking Table Tests to Investigate Soil Desaturation as a Liquefaction Countermeasure. En Seismic Performance and Simulation of Pile Foundations in Liquefied and Laterally Spreading Ground (pp. 282-293). Reston, EE. UU.: American Society of Civil Engineers. DOI: https: / / doi .org/10.1061/40822 (184) 23 Ver página 145

[Okamura y Tomida, 2015] Okamura, M. y Tomida, Y. (2015). Full scale test on cost effective liquefaction countermeasure for highway embankment. En Proceedings of Sixth Internal Geotechnical Sym- 
posium on Disaster Mitigation in Special Geoenvironment Conditions. IIT Madras Chennai, India. $\uparrow$ Ver página 143

[Okamura, Ishihara y Tamura, 2003] Okamura, M., Ishihara, M. y Tamura, K. (2003). Liquefaction resistances and degree of saturation of sand improved with sand compaction piles. En 13th World Conference on Earthquake Engineering (vol. 43, pp. 175-187). DOI: https://doi .org/10.3208/ sandf.43.5_175 个Ver página 142, 147

[Okamura, Ishihara y Tamura, 2006] Okamura, M., Ishihara, M. y Tamura, K. (2006). Degree of saturation and liquefaction resistances of sand improved with sand compaction pile. Journal of Geotechnical and Geoenvironmental Engineering, 132(2), 258-264. DOI: https://doi.org/10.1061/ (ASCE) 1090-0241 (2006) 132:2 (258) 个Ver página 145, 147

[Okamura et al., 2009] Okamura, Mitsu, Takebayashi, M., Nishida, K., Fujii, N., Jinguji, M., Imasato, T., ..., Nakagawa, E. (2009). In-situ test on desaturation by air injection and its monitoring. Japanese.). Journal of Japan Society of Civil Engineers, 65, 756-766. https: / / doi . org/10 . 3850/GI100 个Ver página 145, 147, 148

[Okamura et al., 2011] Okamura, M., Takebayashi, M., Nishida, K., Fujii, N., Jinguji, M., Imasato, T., ..., Nakagawa, E. (2011). In-situ desaturation test by air injection and its evaluation through field monitoring and multiphase flow simulation. Journal of Geotechnical and Geoenvironmental Engineering, 137(7), 643-652. DOI: https: / / doi .org/10.1061/ (ASCE) GT.1943-5606.0000483 $\uparrow$ Ver página $143,147,148$

[PDE Solutions Inc., 2005] PDE Solutions Inc. (2005). FlexPDE Users Guide. Spokane Valley, EE. UU. Recuperado de http: //Www.pdesolutions.com $\uparrow$ Ver página 142

[Pinder y Gray, 2008] Pinder, G. F. y Gray, W. G. (2008). Essentials of multiphase flow in porous media. Hoboken, EE. UU.: Wiley. Recuperado dehttps://books.google.com.co/books?id= V3srAMAzw-EC https://doi.org/10.1002/9780470380802 个er página 149

[Pruess, 1991] Pruess, K. (1991). TOUGH2: A general-purpose numerical simulator for multiphase nonisothermal flows. Berkeley, EE. UU. DOI: https: // doi .org/10.2172/138333 ^Ver página 148

[Rad, Vianna y Berre, 1994] Rad, N. S., Vianna, A. J. D. y Berre, T. (1994). Gas in soils. II: Effect of gas on undrained static and cyclic strength of sand. Journal of Geotechnical Engineering, 120(4), 716- 736. DOI:https://doi.org/10.1061/(ASCE) 0733-9410(1994)120:4 (716) 个Ver página 143

[Ramos, 2015] Ramos, A. M. (2015). Influence of the void ratio and the confining on the static liquefaction in slopes in changi sand. Tecnura, 19(43). 57-67. DOI: https: / / doi . org/10.14483/ udistrital.jour.tecnura.2015.1.a04 个er página 149 
[Ramos, Prada y Vega, 2016] Ramos, A. M., Prada, L. F. y Vega, C. A. (2016). Análisis de elementos finitos con un continuo elástico lineal tipo Cosserat. Tecnura 2016.4.a03 20(50). 43-54. DOI: https : //doi.org/10.14483/udistrital.jour.tecnura. $\uparrow$ Ver página 149

[Reddy y Adams, 2001] Reddy, K. R. y Adams, J. A. (2001). Effects of soil heterogeneity on airflow patterns and hydrocarbon removal during in situ air sparging. Journal of Geotechnical and Geoenvironmental Engineering, 127(3), 234-247. DOI: https://doi.org/10.1061/(ASCE) 1090-0241 (2001) 127:3 (234) 个Ver página 146

[Reddy, Kosgi y Zhou, 1995] Reddy, K. R., Kosgi, S. y Zhou, J. (1995). A review of in-situ air sparging for the remediation of VOC-contaminated saturated soils and groundwater. Hazardous Waste and Hazardous Materials, 12(2), 97-118. DOI: https://doi.org/10.1089/hwm.1995.12.97 个Ver página 147

[Takemura et al., 2009] Takemura, J., Igarashi, R., Izawa, J., Okamura, M. y Masuda, M. (2009). Centrifuge model tests on soil desaturation as a liquefaction countermeasure. En Proceedings of the 17th International Conference on Soil Mechanics and Geotechnical Engineering: The Academia and Practice of Geotechnical Engineering (vol. 1, pp. 502-505). DOI: https://doi.org/10.3233/ 978-1-60750-031-5-502 $\uparrow$ Ver página 148

[Tomida, 2014] Tomida, Y. (2014). A study on soil desaturation as a liquefactioncounter- measure for highway embankmens. Japón: Ehime University. $\uparrow$ Ver página 143, 146

[Tsukamoto, Kawabe, Matsumoto y Hagiwara, 2014] Tsukamoto, Y., Kawabe, S., Matsumoto, J. y Hagiwara, S. (2014). Cyclic resistance of two unsaturated silty sands against soil liquefaction. Soils and Foundations, 54(6), 1094-1103. DOI: https : / doi.org/10.1016/j.sandf.2014.11.005 个Ver página 142

[Tsukamoto et al., 2002] Tsukamoto, Y., Ishihara, K., Nakazawa, H., Kamada, K. y Huang, Y. (2002). Resistance of partly saturated sand to liquefaction with reference to longitudinal and shear wavevelocities. Soils and Foundations, 42(6), 93-104. DOI: https: / / doi . org/10 .3208/sandf. 42. 6_93 $\uparrow$ Ver página 142

[US EPA, 1992] United States Environmental Protection Agency (US EPA) (1992). A technology assessment of soil vapor extraction and air sparging. EPA/600/R-92/173. Cincinnati, EE. UU. $\uparrow$ Ver página 145, 146

[Van Genuchten, 1980] Van Genuchten, M. T. (1980). A closed-form equation for predicting the hydraulic conductivity of unsaturated soils. Soil Science Society of America Journal, 44(5), 892. DOI: https://doi.org/10.2136/sssaj1980.03615995004400050002x 个Ver página 151 
[Vega, Ramos y García, 2017] Vega, C. A., Ramos, A. M. y García, E. F. (2017). Efecto del gas en la velocidad de onda de corte de suelos arenosos densificados con explosivos. Tecnura, 21(51), 67-80. DOI: https://doi.org/10.14483/udistrital.jour.tecnura.2017.1.a05 个Ver página 145

[Wisconsin DNR, 1995] Wisconsin DNR (1995). Updated information and errata regarding guidance on design, installation and operation of in situ air sparging systems. File Ref. 4440. Madison, EE. UU. $\uparrow$ Ver página 145

[Yang, Savidis y Roemer, 2004] Yang, J., Savidis, S. y Roemer, M. (2004). Evaluating liquefaction strength of partially saturated sand. Journal of Geotechnical and Geoenvironmental Engineering, 130(9), 975-979. DOI: https: / / doi.org/10.1061/(ASCE) 1090-0241 (2004)130:9 (975) 个Ver página 142

[Yang, Savidis, Sato y Li, 2003] Yang, J, Savidis, S., Sato, T. y Li, X. S. (2003). Influence of vertical acceleration on soil liquefaction: new findings and implications. Proceeding Soil and Rock America, 1. $\uparrow$ Ver página 143

[Yashima, Oka, Taguchi y Tateishi, 1995] Yashima, A., Oka, F., Taguchi, Y. y Tateishi, A. (1995). Three dimensional liquefaction analysis considering the compressibility of fluid phase. En Proceedings 40th JGS Symposium (pp. 257-264). $\uparrow$ Ver página 148

[Yasuhara, Okamura y Kochi, 2008] Yasuhara, H., Okamura, M. y Kochi, Y. (2008). Experiments and predictions of soil desaturation by air-injection technique and the implications mediated by multiphase flow simulation. Soils and Foundations, 48(6), 791-804. DOI: https : / / doi . org/ 10 . 3208 /sandf . 48 . 791 个Ver página 147, 148, 150,151,152,153, 157

[Yegian, Eseller-Bayat, Alshawabkeh y Ali, 2007] Yegian, M. K., Eseller-Bayat, E., Alshawabkeh, A. y Ali, S. (2007). Induced-partial saturation for liquefaction mitigation: experimental investigation. Journal of Geotechnical and Geoenvironmental Engineering, 133(4), 372-380. DOI: https : / / do i . org / $10.1061 /$ (ASCE) 1090-0241 (2007)133:4(372) 个Ver página 142

[Yoshimi, Tanaka y Tokimatsu, 1989] Yoshimi, Y., Tanaka, K. y Tokimatsu, K. (1989). Liquefaction resistance of a partially saturated sand. Soils and Foundations, 29(3), 157-162. DOI: https: / / doi . org/10.3208/sandf1972.29.3_157 个Ver página 155

[Zeybek y Madabhushi, 2017a] Zeybek, A. y Madabhushi, G. S. P. (2017a). Centrifuge testing to evaluate the liquefaction response of air-injected partially saturated soils beneath shallow foundations. Bulletin of Earthquake Engineering, 15(1), 339-356. DOI: https://doi.org/10.1007/

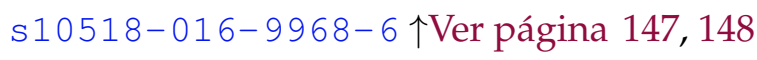


[Zeybek y Madabhushi, 2017b] Zeybek, A. y Madabhushi, G. S. P. (2017b). Influence of air injection on the liquefaction- induced deformation mechanisms beneath shallow foundations. Soil Dynamics andEarthquake Engineering, 97, 266-276. DOI: https://doi.org/10.1016/j. soildyn.2017. $03.018 \uparrow$ Ver página 147, 148

[Zeybek y Madabhushi, 2017c] Zeybek, A. y Madabhushi, G. S. P. (2017c). Durability of partial saturation to counteract liquefaction. Proceedings of the Institution of Civil Engineers: Ground Improvement, 170(2),102-111. DOI: https://doi.org/10.1680/jgrim.16.00025 个Ver página 145

[Zeybek y Madabhushi, 2018] Zeybek, A. y Madabhushi, G. S. P. (2018). Physical modelling of air injection to remediate liquefaction. International Journal of Physical Modelling in Geotechnics, 18(2), 68-80. DOI: https: //doi.org/10.1680/jphmg.16.00049 Ver página 147, 148 